\title{
Effect of maternal anticonvulsant treatment on neonatal blood coagulation
}

\author{
Edmund Hey
}

\begin{abstract}
Aims-To investigate the impact of maternal anticonvulsant use on the ability of cord blood to coagulate.

Methods-Cord blood prothrombin times were measured, over 15 years in a consecutive series of 137 term babies born to women taking phenobarbitone, phenytoin, and/or carbamazepine while pregnant. The response to parenteral vitamin $K$ was measured in 83 neonates.

Results-Only 14 of the 105 babies born to the mothers who had therapeutic anticonvulsant blood concentrations at birth had a prolonged prothrombin time (outside the $95 \%$ reference range). None had an overt bleeding tendency. The abnormality was corrected within 2 hours by $1 \mathrm{mg}$ of parenteral vitamin $K$, but rapid intravenous prophylaxis produced complications in three infants.

Conclusions-A policy of giving vitamin $\mathrm{K}$ throughout the last third of pregnancy to all women being treated with anticonvulsants, as recently recommended, is not justified by the available evidence. The belief that there is a distinct, early form of neonatal vitamin $K$ deficiency that is different from, and more dangerous than, the classic form of the disease, is not supported by a review of the published evidence.

(Arch Dis Child Fetal Neonatal Ed 1999;81:F208-F210)
\end{abstract}

Keywords: anticonvulsant medication in pregnancy; early vitamin $\mathrm{K}$ deficiency bleeding; prothrombin deficiency

Forty years have elapsed since the first published report suggested that maternal anticonvulsant treatment might increase the vulnerability of a neonate to a form of coagulopathy-haemorrhagic disease of the newborn - correctable with vitamin $\mathrm{K} .{ }^{1}$ However, the problem was generally considered rare until a study in 1970 which reported a high incidence of prothrombin deficiency among babies born to mothers taking anticonvulsant medication. ${ }^{2}$ This prompted a 15 year study of the coagulation status of all such babies in Newcastle using blood samples collected from the mother, and from the umbilical cord, at delivery. Because of a fear that intramuscular administration of vitamin $\mathrm{K}$ might itself cause bleeding, these babies were also admitted briefly to special care for the first 10 years of that study so they could be given vitamin K (1 $\mathrm{mg}$ of Konakion) intravenously, and further blood specimens were collected without trauma through an umbilical vein catheter 2-4 hours later. A review of this policy in 1979 revealed that significant prolongation of the prothrombin time was relatively rare, and umbilical catheterisation not without hazard, so a policy of immediate intramuscular prophylaxis was substituted.

In the light of the renewed debate about the indications for oral and intramuscular prophylaxis, the experience assembled during this previously unpublished study is now reported.

\section{Methods}

Blood was collected with a needle and syringe from the umbilical cord of 137 term babies whose mothers had been taking anticonvulsant drugs throughout pregnancy (care being taken to prevent contamination with Wharton's jelly) and $2.5 \mathrm{ml}$ of this blood added to $0.25 \mathrm{ml}$ of $3.8 \%$ sodium citrate. The one stage prothrombin time was then measured on the spun sample using a rabbit brain thromboplastin (Dade, American Hospital Supply Corporation) from 1970 to 1980 , and a human brain thromboplastin (Manchester reagent, Thrombosis Reference Centre, Manchester) thereafter. Citrated control plasma was obtained by pooling the plasma from 10 adult volunteers, and the prothrombin time expressed as a ratio of the time it took a simultaneous control plasma to clot. Blood was also collected from all but six of the mothers at delivery to determine anticonvulsant concentrations. Eighty three of the babies had a further specimen collected from an umbilical vein catheter 2 hours after receiving $1 \mathrm{mg}$ of intravenous vita$\min \mathrm{K}$.

Cord blood was collected during the first year of the study from 50 term babies whose mothers had not received any antenatal treatment, and from a further 50 control babies during the final year. The distribution of these ratios was normal after log transformation, and the two means and standard deviations were almost identical despite the change in thromboplastin two thirds of the way through the study.

\section{Results}

All the mothers had been taking phenobarbitone, phenytoin, or carbamazepine, or some combination of the three, but nearly a quarter (32/137) had anticonvulsant blood concentrations less than half the lower limit of the normally quoted therapeutic range at delivery, and subsequent enquiry revealed that many mothers had been taking treatment since childhood without medical review even though they had been free from seizures for five or even 10 years. 


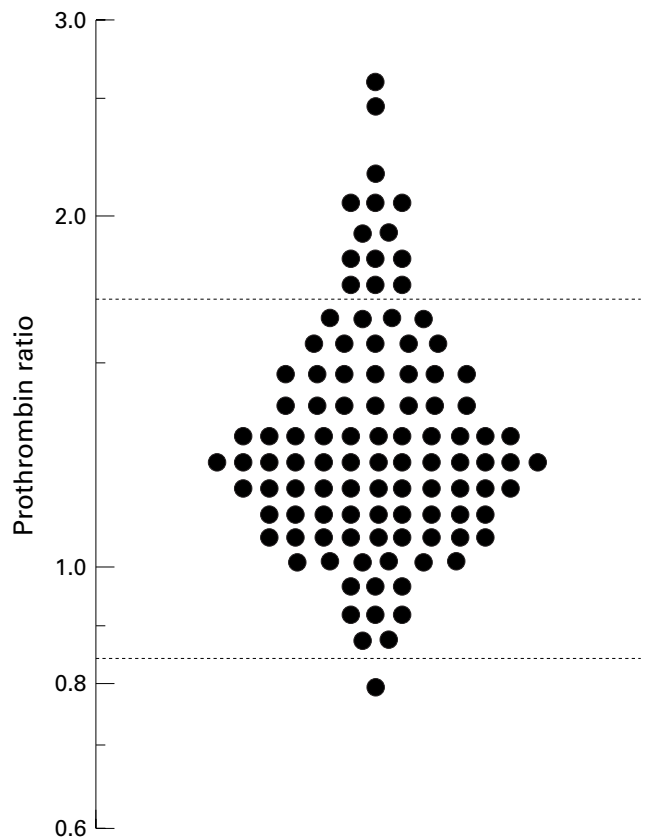

Figure 1 Cord blood prothrombin ratio in 105 babies whose mothers probably had therapeutic blood anticonvulsant concentrations at delivery. The reference range (within which $95 \%$ of the control ratios lay) is shown by the dotted lines. Although the mean ratio is higher in the babies of mothers receiving anticonvulsant treatment, it is the tail of high values that is of clinical significance.

The mean prothrombin ratio for the 105 babies born to mothers receiving effective anticonvulsant treatment was higher than in the control babies, but only 14 had a prothrombin ratio above the $95 \%$ reference range for control babies (fig 1). There was no correlation between the initial neonatal prothrombin ratio and the amount of maternal medication taken, and no change in the mean ratio over time. The prothrombin ratio changed little in most of the babies after vitamin $\mathrm{K}$ administration, but it fell at least $25 \%$ in all 10 of the 68 with an initial cord blood ratio in excess of 1.7 who had been given intravenous vitamin $\mathrm{K}$ at birth. No baby had any overt evidence of a bleeding tendency at birth, none bled from any intramuscular injection site, and none developed clinical evidence of vitamin $\mathrm{K}$ deficiency later.

Nevertheless, three of the 83 babies admitted to special care for 4-6 hours at birth so that vitamin $\mathrm{K}$ could be given through a temporarily sited umbilical vein catheter, developed complications that could have been a result of this management. One baby (with a prothrombin ratio of 1.2) had a $30 \mathrm{ml}$ bleed from the umbilical stump one hour after the catheter was withdrawn, and one baby required surgery for a focal colonic perforation on the second day of life. A third developed listeria infection after being nursed next to a baby with undiagnosed congenital listeria in the nursery for two hours.

\section{Discussion}

Overt bleeding due to vitamin $\mathrm{K}$ deficiency is a rare but well described phenomenon in babies born to mothers taking phenytoin or a barbiturate anticonvulsant for epilepsy. ${ }^{2-4}$

\section{Key messages}

- Few babies born to women taking phenobarbitone, phenytoin and/or carbamazepine have an overt bleeding tendency at birth, although $13 \%$ have a prothrombin time that is longer than normal (above the $95 \%$ reference range)

- A significant number of women seem to be taking subtherapeutic doses of these potentially teratogenic anticonvulsants in an unsupervised (and probably unnecessary) way

- Parenteral treatment at delivery suffices to correct the coagulation abnormality within 2 hours (the adequacy of oral treatment remains undetermined)

Nevertheless, of the 38 published cases, less than a third ${ }^{1-13}$ can be counted as established cases by the criteria most would now judge appropriate in any epidemiological study of this condition. ${ }^{14}$ Many of the more adequately documented cases (with a measured prothrombin time and a response to vitamin $\mathrm{K}$ administration) only had mild symptoms, and half first presented when more than 48 hours old. Only two of these babies developed symptomatic bleeding despite the administration of prophylactic intramuscular vitamin $\mathrm{K}$ at birth: both had sustained trauma to the liver, ${ }^{10}{ }^{12}$ and only one had evidence of a generalised bleeding tendency. ${ }^{10}$ The suggestion that anticonvulsants could depress the fetal vitamin $K$ dependent factor concentrations seriously enough to cause death from bleeding before birth ${ }^{15}$ deserves more study, but remains to be substantiated.

It is now widely held that maternal anticonvulsant treatment leaves babies vulnerable to a distinct early form of vitamin $\mathrm{K}$ deficiency bleeding that is different from, and more lethal than, the classic disease, ${ }^{16}{ }^{17}$ but this is to misread the published case reports. It would be more correct to say that such babies are at greater risk of severe bleeding if they sustain serious trauma during delivery, and they are also more likely to develop classic symptoms later in the first week of life, unless given prophylactic vitamin $\mathrm{K}$ at birth. It was a demonstrable reduction in the number of deaths associated with lethal early intracranial and intraperitoneal bleeding, just as much as a reduction in the number showing evidence of a generalised but less serious bleeding tendency later in the first few days of life, that motivated the Scandinavian paediatricians who first recommended universal (oral) prophylaxis 50 years ago. ${ }^{18-21}$ Units that later adopted a policy of selective intramuscular prophylaxis (often for lack of any licensed oral alternative) were reflecting this double objective. The target was babies who had sustained a traumatic delivery, and babies who were unlikely to get much milk in the first few days of life. The slow transformation of this logical strategy into a policy that resulted in intramuscular vitamin $\mathrm{K}$ being given to every baby having a caesarean or forceps delivery, and every baby going to 
special care, shows what can happen when staff no longer understand the reasons underpinning unit protocol.

Five of the 16 babies born to mothers receiving anticonvulsant treatment in the study reported by Mountain et al had a low cord blood prothrombin (factor II) concentration. Deblay et al encountered bleeding in $7 \%$ (8/115) of babies not given vitamin $\mathrm{K}$ at birth. They also found a significant reduction in mean prothrombin concentration in a prospective study of 74 babies, ${ }^{22}$ and argued that the best way to prevent this was to give the mother additional oral vitamin $\mathrm{K}$. Vitamin $\mathrm{K}$ does not cross the placenta readily, so large doses have to be given. Treatment with $20 \mathrm{mg}$ a day for two weeks before delivery was thought to be associated with a higher prothrombin concentration in the 14 babies born to mothers so treated in this report, but the study was too small for the difference to be statistically convincing. ${ }^{22}$ Treatment with $10 \mathrm{mg}$ every day for a month abolished all evidence of subclinical vitamin $\mathrm{K}$ deficiency in a more recent study of 16 babies, ${ }^{23}$ but prothrombin times were not reported. Maternal prophylaxis does not reduce the cord blood prothrombin time of normal preterm babies. ${ }^{24} 25$

That some babies born to mothers receiving phenobarbitone or phenytoin do have marginally prolonged prothrombin times at birth is confirmed by this study, but symptomatic deficiency of vitamin $\mathrm{K}$ dependent factors was not seen, and seems to be rarer than early reports have suggested. As immediate prophylaxis with intramuscular vitamin $\mathrm{K}$ at birth might well have prevented all the published cases of documented symptomatic factor deficiency unassociated with trauma to the liver or spleen, many clinicians will prefer to give a physiological dose of vitamin $\mathrm{K}$ to the baby at delivery, rather than a pharmacological dose to the mother before delivery, as is frequently recommended, ${ }^{4172326}$ especially while there remains any continuing possibility that high doses could be carcinogenic. ${ }^{27} 28$ The problem should become less common as other drugs come to be used in the management of adult epilepsy-valproate, in particular, does not seem to cause neonatal hypoprothrombinaemia. ${ }^{3}$

This study was initiated 29 years ago by the late Dr Gerald Neligan, with advice and laboratory support from the late Professor William Walker, and undertaken at the Princess Mary Maternity Hospital in Newcastle, which has now closed.

I am grateful to Dr M Reid and Dr M Cornelissen for helpful comment and advice.

1 van Creveld S. Nouveaux aspects de la maladie hémorragique du nouveau-né. Arch Fr Pediatr 1958;15:721-35.
2 Mountain KR, Hirsh J, Gallus AS. Neonatal coagulation defect due to anticonvulsant drug treatment in pregnancy. Lancet 1970;i:265-8.

3 Cornelissen M, Steegers-Theunissen R, Kollée L, et al. Increased incidence of neonatal vitamin $\mathrm{K}$ deficiency resulting from maternal anticonvulsant exposure. $A m \mathcal{F}$ Obstet Gynecol 1993;168:923-7.

4 Åstedt B. Antenatal drugs affecting the vitamin K status of the fetus and the newborn. In: Sutor AH, Hathaway WE, eds. Vitamin K in Infancy. Stuttgart: Schattauer, 1995: 85-91.

5 Douglas $\mathrm{H}$. Haemorrhage in the newborn. Lancet 1966;i:816-7.

5 Alagille D, Odievre M, Houllemare L, Viterbo G, Leroy D. Avitaminose $\mathrm{K}$ neonatale sevère chez 2 enfants nés de mère traitée par des anticomitiaux. Arch Fr Pediatr mère traitée $p$

7 Monnet P, Rosenberg D, Bovier-Lapierre M. Thérapeutique anticomitiale administrée pendant la grossese et maladie hémorragique du nouveau-né. Remarques critiques à propos de trois observations personnelles. Rev Fr Gynecol Obstet 1968;63:695-702.

8 Margolin FG, Kantor NM. Hemorrhagic disease of the newborn. An unusual case related to maternal ingestion of an anti-epileptic drug. Clin Ped 1972;11:59-60.

9 Waltl H, Mitterstieler G, Schwingshackl A. Hämorrhagische diasthese bei einen neugeborenen einer mutter mit antiepilischer therapie. Dtsch Med Wochenschr 1974;99:1315-7.

10 Bleyer WA, Skinner AL. Fatal neonatal hemorrhage after maternal anticonvulsant therapy. $\mathcal{F} A M A$ 1976;235:626-7.

11 Griffiths AD. Neonatal haemorrhage associated with maternal anticonvulsant therapy. Lancet 1981;ii:1296-7.

12 Srinivasan G, Seeler RA, Tiruvury A, Pildes RS. Maternal anticonvulsant therapy and hemorrhagic disease of the newborn. Obstet Gynecol 1982:59:250-2.

13 McNinch AW, Orme R L-E, Tripp JH. Haemorrhagic disease of the newborn returns. Lancet 1983;i:1089-90.

14 Tripp J, Cornelissen M, Loughnan P, McNinch A, Schubiger G, von Kries R. Suggested protocol for the reporting of prospective studies of vitamin $\mathrm{K}$ deficiency reporting of prospective studies of vitamin $K$ deficiency bleeding (previously called hemorrhagic disease of the newborn). In: Sutor AH, Hathaway WE, eds.
infancy. Stuttgart: Schattauer, 1995: 395-401.

15 Speidel BD, Meadow SR. Maternal epilepsy and abnormalities of the fetus and newborn. Lancet 1972;ii:839-43.

16 Lane PA, Hathaway WE. Vitamin $\mathrm{K}$ in infancy. $\mathcal{F}$ Pediatr 1985;106;351-9.

17 Sutor AH. Vitamin K deficiency bleeding in infancy: a status report. In: Sutor AH, Hathaway WE, eds. Vitamin K in Infancy. Stuttgart: Schattauer, 1995: 3-18.

18 Salomonsen L. Morbus hemorrhagicus neonatorum. (Hypoprothrombinemia neonatorum). Acta Paediatr Scand poprothrombinemia neonatc

19 Lehman, J. Vitamin K as a prophylactic in 13,000 infants. Lancet 1944;1:493-4.

20 Anonymous. Haemorrhage in the newborn. [Editorial]. Lancet 1944;i:506-8.

21 Lehmann J. Vitamin $\mathrm{K}$ as a prophylactic. Lancet 1944;ii:737-8.

22 Deblay MF, Vert P, Andre M, Marchal F. Transplacental vitamin $\mathrm{K}$ prevents haemorrhagic disease of infants of epileptic mothers. Lancet 1982;i:1247.

23 Cornelissen M, Steegers-Theunissen R, Kollée L, Eskes T, Motohara K, Monnens L. Supplementation of vitamin K in pregnant women receiving anticonvulsant therapy in pregnant women receiving anticonvulsant therapy prevents neonatal vi
col $1993 ; 168: 884-8$.

24 Kazzi NJ, Ilagan NB, Liang K-C, et al. Maternal administration of vitamin $\mathrm{K}$ does not improve the coagulation profile of preterm infants. Pediatrics 1989;84:104550.

25 Thorp JA, Parriot J, Ferrette-Smith D, Meyer BA, Cohen GR, Johnson J. Antepartum vitamin $\mathrm{K}$ and phenobarbital for preventing intraventicular hemorrhage in the premature infant: a randomised, double-blind, placebocontrolled study. Obstet Gynecol 1994;83:70-6.

26 Wallace H, Shorvon SD, Hopkins A, O'Donoghue M. Adults with poorly controlled epilepsy. London: Royal College of Physicians, 1997.

27 Passmore SJ, Draper G, Brownbill P, Kroll M. Case-control studies of relation between childhood cancer and neonatal vitamin K administration. BMF 1998;316:178-84

28 Tripp JH, McNinch AW. The vitamin K debacle: cut the Gordian knot but first do no harm. Arch Dis Child 1998;79:295-7. 